\title{
A NEW SIGNATURE PROTOCOL BASED ON RSA AND ELGAMAL SCHEME
}

\author{
J. Ettanfouhi and O. Khadir \\ Laboratory of Mathematics, Cryptography and Mechanics, Fstm, \\ University Hassan II of Casablanca, Morocco
}

\section{ABSTRACT}

In this paper, we present a new signature scheme based on factoring and discrete logarithm problems. Derived from a variant of ElGamal signature protocol and the RSA algorithm, this method can be seen as an alternative protocol if known systems are broken.

\section{KEYWORDS}

Factoring, DLP, PKC, ElGamal signature scheme, RSA.

MSC: $94 A 60$

\section{INTRODUCTION}

In 1977, Rivest, Shamir, and Adleman[6] described the famous RSA algorithm which is based on the presumed difficulty of factoring large integers. In 1985, ElGamal [2] proposed a signature digital protocol that uses the hardness of the discrete logarithm problem[ 5 p. 116,7 p. 213,8 p. 228 ]. Since then, many similar schemes were elaborated and published[1,3].

Among them, a new variant was conceived in 2010 by the second author[ 4 ].In this work, we apply a combination of the new variant of Elgamal and RSA algorithm to build a secure digital signature. The efficiency of the method is discussed and its security analyzed.

The paper is organised as follows: In section 2, we describe the basic ElGamal digital signature algorithm and its variant. Section 3 is devoted to our new digital signature method. We end with the conclusion in section 4 .

In the paper, we will respect ElGamal work notations [3]. N, Z are respectively the sets of integers and non-negative integers. For every positive integer $n$, we denote by $Z / n Z$ the finite ring of modular integers and by $(Z / n Z)^{*}$ the multiplicative group of its invertible elements. Let $a, b, c$ be three integers. The GCD of $a$ and $b$ is written as $\operatorname{gcd}(a, b)$. We write $a \equiv b \quad 0.1 \mathrm{~cm}[c]$ if $c$ divides $a-b$, and $a=b \bmod c$ if $a$ is the rest in the division of $b$ by $c$. The bit length of $n$ is the number of bits in its binary model, with $n$ an integer.We start by presenting the basic ElGamal digital signature algorithm and its variant: 


\section{ELGAMAL SIGNATURE SCHEME}

In this section we recall ElGamal signature scheme[2] and its variant[4].

1. Alice chooses three numbers:

- $p$, a large prime integer.

- $\alpha$, a primitive root of the finite multiplicative group $(\mathrm{Z} / \mathrm{pZ})^{*}$

- $x$, a random element of $\{1,2, \ldots, p-1\}$

2. She computes $y=\alpha^{x} \bmod p$. Alice's public key is $(p, \alpha, y)$, and $x$ is her private key.

3. To sign the document $m$, Alice must solve the problem:

$$
\alpha^{m} \equiv y^{r} r^{s}[p]
$$

where $r, s$ are the unknown variables.

Alice fixes arbitrary $r$ to be $r=\alpha^{k} \bmod p$, where $k$ is chosen randomly and invertible modulo $p-1$. Equation (1) is then equivalent to:

$$
m \equiv x r+k s \quad[p-1]
$$

Since Alice has the secret key $x$, and as the number $k$ is invertible modulo $p-1$, she calculates the other unknown variable $s$ by

$$
s \equiv \frac{m-x r}{k} \quad[p-1]
$$

4. Bob can verify the signature by checking if congruence (1) is valid for the variables $r$ and $s$ given by Alice.

\section{VARIANT OF ELGAMAL SIGNATURE SCHEME}

We present a variant of ElGamal digital signature system.

This variant Error! Reference source not found. is based on the equation:

$$
\alpha^{t} \equiv y^{r} r^{s} s^{m}[p]
$$

$r, s, t$ are the unknown parameters, and $(p, \alpha, y)$ are Alice public keys. $p$ is an integer (a large prime). $\alpha$ is a primitive root of $Z_{p}^{*} . y$ is calculated by $y=\alpha^{x} \bmod p . x$ is a random element of $\{1,2, \ldots, \mathrm{p}-1\}$. 
Let $m=h(M)$, where $h$ is a hash function, and $\mathrm{M}$ the message to be signed by Alice.

To give the solution (3), she fixes randomly $r$ as $r \equiv \alpha^{k} \bmod p$, and $s$ to be $s \equiv \alpha^{l} \bmod p$, where $k, l$ are selected arbitrary in $\{1,2, \ldots, \mathrm{p}-1\}$.

Equation (3) is then equivalent to:

$$
t \equiv r x+k s+\operatorname{lm}[p-1]
$$

as Alice recognize the values of $r, s, k, l, m, x$, she is able to calculate the last unknown variable $t$.

Bob verify the signature by verifying the congruence (4).

this system does not use the extended Euclidean algorithm for calculating $k^{-1} \bmod (p-1)$.

We clarify the scheme by the example given by the creator of this alternative[4].

\subsection{EXAMPLE}

Let $(p, \alpha, y)$ be Alice public keys where: $p=509, \alpha=2$ and $y=482$. We assert that we are not confident if using a small value of $\alpha$ does not abate the protocol. The private key is $x=281$. Suppose that Alice wants to generate a signature for the document $M$ for which $m \equiv h(M) \equiv 432$ [508] with the exponents $k=208$ and $l=386$ are randomly taken. She computes $r \equiv \alpha^{k} \equiv 2^{208} \equiv 332[p], s \equiv \alpha^{l} \equiv 2^{386} \equiv 39[p]$ and $t \equiv r x+k s+\operatorname{lm} \equiv 440[p-1]$.

Bob or anyone can verify the relation $\alpha^{t} \equiv y^{r} r^{s} s^{m}[p]$. Indeed, we find that $\alpha \equiv 436[p]$ and $y^{r} r^{s} s^{m} \equiv 436[p]$.

\section{OUR PROTOCOL}

\subsection{DESCRIPTION}

In this section, we describe our new digital signature. The protocol is based simultaneously on two hard problems.

We assume first that $h$ is a public secure hash function like SHA1[5 p.348, 7 p. 242,8 p.133].

We suppose that Alice public keys are $(P, \alpha, y, e)$ where:

- $P=2 p q+1, p, q$ are three primes.

- $\alpha$, a primitive root of the multiplicative group $(\mathrm{Z} / p \mathrm{Z})^{*}$.

- $y=\alpha^{x} \bmod P$, where $x$ is the private key of Alice, which is randomly taken in $\{1,2, \ldots, P-1\}$. 
- Element $e$ is the public exponent in the RSA cryptosystem.

We propose the following protocol:

If Alice wants to sign the message $M$, she must give a solution for the modular equation:

$$
\alpha^{t} \equiv y^{r^{e}}\left(r^{e}\right)^{\left(s^{e}\right)}\left(s^{e}\right)^{m}[P]
$$

where $m=h(M) \bmod p$, and $r, s, t$ are unknown.

To solve equation (5), Alice starts by putting:

$$
\begin{gathered}
r^{\prime} \equiv r^{e} \quad[P-1] \\
s^{\prime} \equiv s^{e} \quad[P-1]
\end{gathered}
$$

Equation (5) becomes:

$$
\alpha^{t} \equiv y^{r} r^{\prime s} s^{\prime m} \quad[P]
$$

Alice uses the new variant of Elgamal algorithm[4] to solve equation (9) and to get the values of $r^{\prime}, s^{\prime}$ and $t$.

Then with her RSA private key she solves equations (7) and (8). The cupel $r$ and $s$ is her signature for the message $M$.

Bob or anybody can check that the signature is valid by replacing $r, s$ and $t$ in relation (5).

\subsection{EXAMPLE}

Let us illustrate the method by the following example.

Suppose that Alice's public key is: $P=2 * 167 * 313+1=104543, \alpha=5, y=23292, e=7$.

The private keys for RSA and ElGamal systems are respectively: $x=9502, d=7399$.

Assume that $m=h(M)=12345$ is the hashed message that she likes to sign.

If she takes Randomly $k=845$ and $l=2561$.

She will find from equation 8 that $r^{\prime}=17744, s^{\prime}=31839$.

Relation 4 implies $t=57764$.

Alice uses (6) and (7) to obtain: 
International Journal of Information Technology, Modeling and Computing (IJITMC) Vol. 4, No.3, August 2016

$$
\begin{array}{ll}
r \equiv r^{\prime d} & {[P-1] \equiv 75282} \\
s \equiv s^{\prime d} & {[P-1] \equiv 19005 .}
\end{array}
$$

To verify Bob puts $A=\alpha^{t} \bmod P=62833, \quad B=y^{r^{e} \bmod P-1} \bmod P=79849$, $C=\left(r^{e} \bmod P-1\right)^{\left(s^{e} \bmod P-1\right)} \bmod P=83421$ and $D=\left(s^{e} \bmod P-1\right)^{m} \bmod P=212997$, and checks if $A=B * C * D \bmod P$.

\subsection{SECURITY ANALYSIS}

Now that we have presented the protocol, we will discuss some possible attacks. Assume that Oscar is Alice's opponent.

ATTACK 1: If the attacker try to imitate the computation made by Alice, he can find $r$ and $s$, but to find $t$ he needs the value of the private key $x$ to solve equation 4 .

ATTACK 2: Suppose Oscar is capable to solve the discrete logarithm problem [2]. He cannot calculate $r$ and $s$ from equation (7) and (8) he will be confronted to the factorisation of a large composite modulus [5,8].

ATTACK 3: Suppose Oscar is capable to solve RSA equations (7) and (8). Oscar cannot get $t$ from equation (9) since $x$ is Alice's secret key. If he tries to get $t$ from equation (4), he will be stopped by the discrete logarithm problem.

\subsection{COMPLEXITY OF OUR ALGORITHM}

As in [1], let $T_{\text {exp }}, T_{m u l t}$ and $T_{h}$ be appropriately the time to calculate an exponentiation, a multiplication and hash function of a document $M$. We neglect the time needed for modular substraction, additions, comparisons and apply the conversion $T_{\text {exp }}=240 T_{\text {mult }}$.

\subsubsection{SIGNATURE COMPLEXITY}

To sign the message $M$, Alice must compute the six parameters:

$m=h(M) \bmod \quad P, \quad r^{\prime} \equiv \alpha^{k} \quad[P], \quad s^{\prime} \equiv \alpha^{l} \quad[P], \quad r \equiv r^{\prime d} \quad[P-1], \quad s \equiv s^{\prime d} \quad[P-1]$, $t \equiv x r^{\prime}+k s^{\prime}+\operatorname{lm}[P-1]$.

Alice needs to perform four modular exponentiations, three modular multiplications and one hash function computation. So the global required time is :

$$
T_{1}=4 T_{\text {exp }}+3 T_{\text {mult }}+T_{h}=963 T_{\text {mult }}+T_{h}
$$

\subsubsection{VERIFICATION COMPLEXITY}

Bob should calculate 4 exponentiations, 2 multiplications and one hash function. So the global required time is :

$$
T_{2}=4 T_{\text {exp }}+2 T_{m u l t}+T_{h}=962 T_{m u l t}+T_{h}
$$




\section{CONCLUSION}

In this work, we proposed a new signature protocol that can be an alternative if old systems are broken. Our method is based simultanyously on RSA cryptosystem and DLP.

\section{ACKNOWLEDGEMENTS}

This work is supported by the MMS e-orientation project.

\section{REFERENCES}

[1] R. R. Ahmad, E. S. Ismail,and N. M. F. Tahat, A new digital signature scheme based on factoring and discrete logarithms , J. of Mathematics and Statistics (4): (2008), pp. .

[2] T. ElGamal, A public key cryptosystem and a signature scheme based on discrete logarithm problem, IEEE Trans. Info. Theory, IT-31, (1985), pp. .

[3] L.C. Guillou, J.J. Quisquater, A Paradoxial Identity-based SIgnature Scheme Resulting from ZeroKnowledge, Advances in cryptography, LNCS 403, (1990) pp. .

[4] O. Khadir, New variant of ElGamal signature scheme, Int. J. Contemp. Math. Sciences, Vol. 5, no. 34, (2010), pp. .

[5] A. J. Menezes, P. C. van Oorschot and S. A. Vanstone, Handbook of applied cryptography, CRC Press, Boca Raton, Florida, 1997.

[6] R. Rivest, A. Shamir and L. Adeleman, A method for obtaining digital signatures and public key cryptosystems, Communication of the ACM, Vol. no 21, (1978), pp. .

[7] J. Buchmann, Introduction to Cryptography,(Second Edition), Springer 2000.

[8] D. R. Stinson, Cryptography, theory and practice, second Edition, Chapman \& Hall/CRC, 2006.

\section{Authors}

Jaouad Ettanfouhi holds an engineer degree in Computer Science from the University of Hassan II of Casablanca (2011). Member of the laboratory of Mathematics, Cryptography and Mechanics, he is preparing a thesis in public key cryptography.

Dr Omar Khadir received his Ph.D. degree in Computer Science from the University of Rouen, France (1994). Co-founder of the Laboratory of Mathematics, Cryptography and Mechanics at the University of Hassan II Casablanca, Morocco, where he is a professor in the Department of Mathematics. He teaches cryptography for graduate students preparing a degree in computer science. His current research interests include public key cryptography, digital signature, primality, factorisation of large integers and more generally, all subjects connected to the information technology.
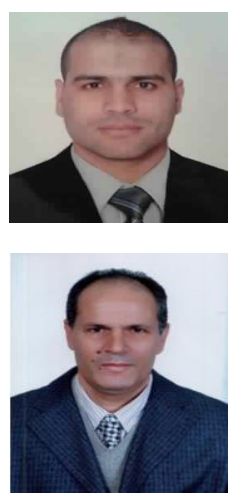